\title{
Recarga de acuíferos con la construcción de qochas en cabecera de cuencas
}

\section{Recharge of aquifers with the construction of qochas in the headwaters of the basins}

\author{
a Mallma Capcha, Tito \\ Facultad de Ingeniería Civil / Universidad Nacional del Centro del Perú \\ Email:tmallma@uncp.edu.pe
}

\section{Resumen}

La recarga del acuífero es importante para poder mantener constante los caudales aguas abajo en los ríos y manantiales; para ello, se han ubicados las cabeceras de cuencas de las subcuecas de los ríos Shullcas, Cunas, Yacus, Achamayo y Chanchas, llegándose a identificar áreas y determinando, en ellos, la escorrentía y la infiltración del agua en el suelo, recargando de esta manera el acuífero.

En la cabecera de las cuencas las precipitaciones anuales son altas llegando este a 794,95 mm; sin embargo, por lo deteriorado en que se encuentra las cabeceras de cuenca, las aguas de precipitación que caen sobre dicha superficie se pierden por escorrentía. Con esta técnica planteada, pues gran parte del agua que se pierde por escorrentía es posible lograr almacenarlo en los acuíferos, porque con dicha precipitación, y en una superficie de 1,00 ha, se puede recargar a los acuíferos hasta un volumen de $1,05 \mathrm{~m}^{3}$ de agua.

Dicha recarga se debe a la velocidad de infiltración del agua por las capas de suelo que en este caso se cuenta con un promedio de $14,57 \mathrm{~cm} / \mathrm{hr}$.

Palabras clave: recursos acuíferos, qocha, cabecera de cuenca, infiltración, precipitación fluvial

\begin{abstract}
The recharge of the aquifer is important to keep the flows downstream in the rivers and springs constant, therefor the headwaters of the sub-basins of the rivers Shullcas, Cunas, Yacus, Achamayo and Chanchas have been located identifying areas and determining in them the runoff and the infiltration of the water in the ground, recharging the aquifer in this way.

In the headwaters of the basins the annual precipitations are high reaching 794,95 mm., However due the deteriorated thing in which are the headwaters of basin the waters of precipitation that fall on this surface are lost by runoff. With this technique is possible to store much of the water lost by runoff in aquifers because of this precipitation and over an area of 1,00 ha, the aquifers can be recharged to a volume of $1,05 \mathrm{~m}^{3}$ of water.
\end{abstract}

This recharge is due to the speed of water infiltration by the layers of soil that in this case has an average of $14,57 \mathrm{~cm} / \mathrm{hr}$.

Keywords: aquifer resources, qocha, basin head, infiltration, river precipitation 


\section{Introducción}

Debido al cambio climático, las precipitaciones que se presentan en las cabeceras de las cuencas son anormales, debido a la existencia de lluvias torrenciales de alta intensidade. Esto hace que la mayor parte del agua de lluvia se pierda por escorrentía, lo que dificulta la infiltración para recargar el acuífero.

La oferta de agua, de las diferentes cuencas, se ve disminuida debido a que las recargas en las regiones altas son escasas, afectando el consumo del recurso para el sector agrícola y el consumo humano; además, de afectar el comportamiento del ecosistema de las cuencas.

\section{Métodos y materiales}

Equipo topográfico, materiales cartográficos, anillo infiltrómetro, materiales de escritorio, equipos de cómputo y equipo fotográfico.

\section{Metodología}

Se determinó el coeficiente de escorrentía en las cabeceras de cuencas. Luego se calculó la infiltración del agua en el suelo de las caberas de cuencas. Se cuantificó la cantidad de agua recargada por año en las qochas construidas.

Las qochas se construyeron en base a estructura verde; es decir, el terraplén se construyó con material propio de la zona y se evaluó la capacidad de desbordamiento de las aguas del río.

\section{Resultados}

Situación actual de las cabeceras de cuencas. Las cabeceras de las cuencas se encuentran degradadas por una serie de acciones provocadas por el hombre, como el sobrepastoreo, la quema de los pastizales, cambio del uso de suelos y pérdi- da de los bosques nativos; los cuales afectan grandemente el ecosistema y, por tanto, la escases de los recursos hídricos.

\section{Recarga de acuíferos}

La recarga del agua hacia el acuífero se pueden efectuar de diferentes maneras, a ello se denomina la siembra de agua, el cual consiste en captar el agua de lluvia y provocar la infiltración en el suelo, con esta práctica se lograra incrementar los caudales en los ríos, en los manantiales y hacer que aparezcan más manantiales o simplemente el suelo se mantendrá húmedo logrando la propagación de vegetaciones.

Las qochas, son sistemas de recarga de agua natural con formaciones topográficas de forma hondonada en donde se depositan el agua de lluvia, el mismo que, luego se infiltra al subsuelo. Actualmente, muchas de estas qochas naturales se encuentran degradadas y cuentan con limitaciones con el volumen de agua que se almacena.

\section{Figura 1}

Estado de erosión de cabecera de cuenca

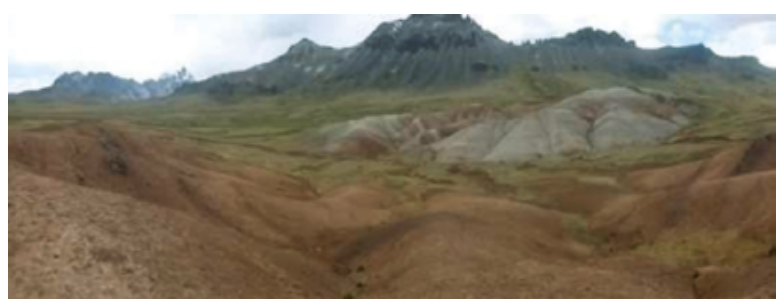

\section{Precipitación en las cabeceras de cuencas}

Las precipitaciones totales anuales en las cabeceras de cuencas son altas al año, por lo es identificado con zona lluviosa, presentándose las precipitaciones intensas durante los meses de noviembre a marzo. El análisis de precipitación, se muestra en la Tabla 1.

\section{Tabla 1}

Precipitación total mensual en cabecera de cuencas $(\mathrm{mm})$.

\begin{tabular}{lcccc}
\hline \multirow{1}{*}{ Estación } & \multicolumn{2}{c}{ Precipitación $(\mathbf{m m})$} & \multicolumn{2}{c}{ Ubicación } \\
\cline { 2 - 5 } & Mínima & Máxima & $\begin{array}{c}\text { Altitud } \\
(\mathbf{m s n m})\end{array}$ & Lugar \\
\hline Huancalpi & 594,5 & 2086,5 & 3970 & Vilca - Hvca. \\
Huancavelica & 698,4 & 1514,5 & 3715 & Hvca. - Hvca. \\
Lircay & 608,9 & 1121,9 & 3357 & Angaraes - Hvca. \\
Túnel Cero & 748,5 & 1093,7 & 4512 & Pilpichaca - Hvca. \\
Paucarbamba & 945,5 & 1744,3 & 3000 & Paucarbamba - Hvca. \\
Laive & 610,3 & 1115,9 & 3842 & Yanacancha - Junín \\
Shullcas & 641,8 & 1442,2 & 3510 & Huancayo - Junín \\
Huaytapallana & 613,7 & 936,9 & 4400 & El Tambo - Junín \\
Acopalca & 641,8 & 1142,2 & 3839 & El Tambo - Junín \\
Ingenio & 404,1 & 1292,8 & 3450 & Sta. Rosa de Ocopa - Junín \\
\hline
\end{tabular}




\section{Precipitaciones con altas intensidades}

Las intensidades máximas de precipitación que ocurren en las cabeceras de cuencas son las que provocan las escorrentías altas los cuales causan erosiones y crecidas inesperadas de los ríos. La intensidad de precipitación y precipitación anual, se muestra en la Tabla 2.

\section{Velocidad de infiltración en la recarga}

La velocidad con que el agua almacenada en las qochas se infiltra en el suelo hasta llegar al acuífero viene a ser aquella que se hace constante, el cual varía de acuerdo a la textura del suelo. El rango de infiltración constante, se muestra en la Tabla 3.

Tabla 2

Intensidad de precipitación en cabecera de cuencas $(\mathrm{mm})$

\begin{tabular}{lcccc}
\hline \multicolumn{1}{c}{ Estación } & $\begin{array}{c}\text { Intensidad de } \\
\text { precipitación } \mathbf{( m m} / \mathbf{h r})\end{array}$ & $\begin{array}{c}\text { Precipitación } \\
\text { total anual (mm) }\end{array}$ & Altitud (msnm) & Ubicación \\
\hline Túnel Cero & 16,64 & 860.80 & 4512 & Pilpichaca - Hvca. \\
Paucarbamba & 18,60 & 685.00 & 3000 & Paucarbamba - Hvca. \\
Laive & 13,51 & 821.10 & 3842 & El Tambo - Junín \\
Acopalca & 16,04 & 894.17 & 3839 & El Tambo - Junín \\
Ingenio & 13,14 & 722.60 & 3450 & Sta. Rosa de Ocopa - Junín \\
Huaytapallana & 15,12 & 786.05 & 4400 & El Tambo - Junín \\
\hline
\end{tabular}

Tabla 3

Infiltración constante según textura de suelo

\begin{tabular}{lcc}
\hline Textura de suelo & $\begin{array}{c}\text { Rango de infiltración } \\
\text { constante }(\mathbf{m m} / \mathbf{h})\end{array}$ & $\begin{array}{c}\text { Promedio de infiltración } \\
\text { constante }(\mathbf{m m} / \mathbf{h r})\end{array}$ \\
\hline Arenoso & $25-50$ & 50 \\
Franco Arenoso & $13-75$ & 25 \\
Franco & $7,5-20$ & 12,5 \\
Franco limoso & $2-15$ & 7,5 \\
Arcillo limoso & $0,2-5$ & 2,6 \\
Arcilla & $0,1-1$ & 0,5 \\
\hline
\end{tabular}

\section{Determinación de la velocidad de infiltración constante}

Para determinar la velocidad de infiltración constante, se realiza la prueba de infiltración con el anillo infiltrómetro, tomándose datos de tiempo y lámina de infiltración, con estos datos se han determinado los parámetros de a y $\mathrm{b}$, empleándose para ello la ecuación de regresión.

$$
\begin{gathered}
Y=a+b x \\
b=\frac{n \sum X_{i} Y_{i}-\sum X_{i} Y_{i}}{n \sum X_{i}^{2} \cdot\left(\sum X_{i}\right)^{2}} \\
a=\frac{\sum Y_{i}}{n}-b \frac{\sum X_{i}}{n}
\end{gathered}
$$

Los resultados, se muestran en la Tabla 4.
Tabla 4

Velocidad de infiltración básica en cabeceras de cuenca

\begin{tabular}{cc}
\hline Textura de suelo & Infiltración constante $(\mathbf{c m} / \mathbf{h})$ \\
\hline Shullcas 1 & 13,99 \\
Shullcas 2 & 16,20 \\
Cunas 1 & 13,90 \\
Cunas 2 & 16,33 \\
Yacus 1 & 17,70 \\
Yacus 2 & 14,68 \\
Achamayo 1 & 14,63 \\
Achamayo 2 & 13,27 \\
Chanchas 1 & 12,92 \\
Chanchas 2 & 12,10 \\
Promedio & 14,57 \\
\hline
\end{tabular}


Intensidades de precipitaciones en las cabeceras de cuencas

Debido al cambio climático en los últimos años, el comportamiento de las precipitaciones son con altas intensidades a partir de las 14 horas y, como la superficie del suelo a ese nivel se encuentra depredada, se produce una alta escorrentía, de acuerdo a al ecuación: Ip $>>$ Ii.

Donde: Ip es la intensidad de precipitación $(\mathrm{mm} / \mathrm{hr})$ y Ii es la velocidad de infiltración en el suelo $(\mathrm{mm} / \mathrm{hr})$.

\section{Escorrentía en las cabeceras de cuencas}

La escorrentía, es el recorrido del agua por grave- dad sobre la superficie del suelo, esta escorrentía va depender de la intensidad de la precipitación, la cobertura vegetal del suelo y la capacidad de infiltración en el suelo. La escorrentía superficial, es la escorrentía del agua de lluvia que ha caído al suelo, que no ha sido infiltrado y, por gravedad, se desplaza por la superficie del suelo siguiendo la pendiente del terreno, el cual se expresa como el resultado del coeficiente de escorrentía y la precipitación. La ecuación fue: $\mathrm{Es}=\mathrm{C} \mathrm{x}$ PP. Los coeficientes de escorrentía y la escorrentía, se muestran en la Tabla 5.

\section{Tabla 5}

Coeficiente de escorrentía y escorrentía en diferentes superficies de terreno

\begin{tabular}{|c|c|c|c|c|c|}
\hline \multirow{2}{*}{$\begin{array}{l}\text { Naturaleza de } \\
\text { la superficie }\end{array}$} & \multirow{2}{*}{$\begin{array}{l}\text { Precipitación } \\
\text { media anual } \\
(\mathrm{mm})\end{array}$} & \multicolumn{2}{|c|}{ Topografía (coeficiente) } & \multicolumn{2}{|c|}{ Escorrentía (mm) } \\
\hline & & $\begin{array}{c}\text { Ondulada } \\
S=5 \text { a } 10 \%\end{array}$ & $\begin{array}{c}\text { Inclinada } \\
\mathrm{S}=\mathbf{1 0} \text { a } \mathbf{3 0} \%\end{array}$ & $\begin{array}{c}\text { Ondulada } \\
S=5 \text { a } 10 \%\end{array}$ & $\begin{array}{c}\text { Pend. Inclinada } \\
\mathrm{S}=10 \text { a } 30 \%\end{array}$ \\
\hline Bosques & 794,95 & 0,18 & 0,21 & 143,09 & 166,94 \\
\hline Pastos Naturales & 794,95 & 0,36 & 0,42 & 286,18 & 333,88 \\
\hline Áreas desnudas & 794,95 & 0,80 & 0,90 & 635,96 & 715,46 \\
\hline
\end{tabular}

\section{Volumen de recarga a los acuíferos}

El volumen de recarga de los acuíferos, se ha considerado tomándose en cuenta una superficie de 1.00 ha, con la velocidad de infiltración constante respectiva para un tiempo de 1 mes; para ello, se ha empleado la siguiente ecuación:

$$
\mathrm{Vol}=\mathrm{Ib} \times \mathrm{A} \times \mathrm{T}
$$

Los volúmenes de escorrentía por terreno, se muestran en la Tabla 6.

Tabla 6

Escorrentía en diferentes Superficies de Terreno

\begin{tabular}{lc}
\hline \multicolumn{1}{c}{ Cuenca } & Volumen $\left(\mathbf{m}^{3}\right)$ \\
\hline Shullcas 1 & 1007280 \\
Shullcas 2 & 1166400 \\
Cunas 1 & 1000800 \\
Cunas 2 & 1175760 \\
Yacus 1 & 1274400 \\
Yacus 2 & 1056960 \\
Achamayo 1 & 1053360 \\
Achamayo 2 & 955440 \\
Chanchas 1 & 930240 \\
Chanchas 2 & 871200 \\
\hline Promedio & $\mathbf{1 0 4 9 1 8 4}$ \\
\hline
\end{tabular}

\section{Resultados}

Hay una relación entre la superficie del suelo, la textura del suelo, la pendiente del terreno y la precipitación en la recarga del acuífero.
El almacenamiento del agua, en zonas adecuadas, mediante la construcción de diques con materiales propios de la zona, permiten que el agua se infiltre hasta la napa freática, logrando la recarga hídrica.

Para elegir las zonas donde poder construir la qochas, se debe de tomar en cuenta las superficies con texturas del suelo que es arenoso, franco arenoso y franco; ya que, son los que tienen una mayor velocidad del infiltración constante del agua.

\section{Conclusiones}

- En las cabeceras de cuencas existen una alta precipitación; sin embargo, estos llegan sin dificultad a los riachuelos y ríos por escorrentía, para luego, depositarse en los océanos. Existen superficies de suelo en las cuencas, los cuales cuentan con suelos permeables, que permiten la infiltración del agua; pero, esta infiltración no es posible, porque la pendiente es alta ( $50 \mathrm{a}$ $80 \%$ ) facilitando la pérdida del agua por escorrentía.

- Para evitar, en parte, la pérdida del agua por escorrentía, este se debe de almacenar en zonas hondonadas; para ello, se debe de construir diques en la parte baja. Los diques, deben tener hasta 1,50 m de altura y deberán de ser construidos utilizando materiales propios de la zona como piedra y tierra compactada.

- La velocidad de infiltración constante promedio de las 5 cabeceras de cuencas, viene a ser $14,57 \mathrm{~cm} / \mathrm{h}$. Con dicha velocidad de infiltración, para un tiempo de 1 mes y una superficie de 1,00 ha, se obtiene un volumen de recarga de $1,05 \mathrm{~m}^{3}$. La escorrentía del agua de lluvia en la cabecera de cuenca, varía de 143,09 a 715,46 mm, en superficies como bosques, pastos naturales y áreas desnudas. 


\section{Referencias bibliográficas}

Aparicio, M. F. (1987). Fundamentos de hidrología de superficie. Ed. Limusa. México.

Chow, V. T. (1964). Handbook of applied hydrology. Mac Graw Hill. New York.

Murillo, D. J. (2004). Recarga de acuíferos, evaluación y análisis de condicionantes técnicos y económicos, acuífero aluvial del Bajo Guadalquivir. Tesis Doctoral. Universidad Politécnica de Madrid.

Rushton, K. (1988). Numerial and conceptual models for recharge estimation in arid and semi-arid zones. En Estimation of Natural Grundwater recharge. Boston.

Vásquez, A. L. (2011). Métodos para determinar la recarga en acuíferos. Posgrado en Aprovechamiento de Recursos Hídricos. Universidad Nacional de Medellín.

Schosinsky, N. G. (2007). Cálculo de la recarga potencial de acuíferos mediante un balance hídrico de suelos. Revista Geológica de América Central. Costa Rica.

Walton, W. (1970). Ground wáter resource evaluation. Mc Graw Hill. New York. 